\title{
A protocol to improve analgesia use in the accident and emergency department
}

\author{
S W Goodacre, R K Roden
}

\begin{abstract}
Objective-To assess the use of analgesia in an accident and emergency (A\&E) department and identify shortcomings. Setting-University teaching hospital.

Methods-An audit of patients referred from the $A \& E$ department to orthopaedic fracture clinic $(n=100)$ or for orthopaedic admission $(n=100)$ was carried out to document analgesia use. An analgesia protocol was introduced and analgesia use was reassessed on the same numbers of patients.
\end{abstract}

Results-Prescribing of analgesia was initially poor: $91 \%$ of fracture clinic referrals and $39 \%$ of admissions received no analgesia while in the $A \& E$ department; when given, it was often by inappropriate routes. Introduction of an analgesia protocol significantly improved analgesia use: fracture clinic referrals receiving unsatisfactory analgesia were reduced from $91 \%$ to $69 \% \quad(P<0.001)$. There was a marked increase in the use of intravenous analgesia, from $9 \%$ to $37 \%$ (P<0.001).

Conclusions-Large numbers of patients still receive no analgesia while in the A\&E department. This seems to be a common problem requiring intervention at a national level. The absence of a coordinated approach to improving analgesia provision for acute trauma in the United Kingdom should be addressed urgently.

( A Accid Emerg Med 1996;13:177-179)

Key terms: analgesia; acute trauma; protocol; audit

There have been very few studies to assess the adequacy of analgesia prescribed in accident and emergency (A\&E) departments. Those that have been published ${ }^{\text {eg } 1}$ have shown inadequate provision of analgesia. We aimed to assess the use of analgesia within our department and identify shortcomings. Having done this we devised a protocol for intervention and assessed whether this was successful. We chose acute skeletal injuries as a well defined group of unequivocally painful injuries that could readily be assessed.

\section{Methods}

One hundred consecutive referrals to the fracture clinic and 100 orthopaedic admissions were analysed for the following data: (1) patient's sex and age; (2) injury sustained; (3) analgesia given in $\mathrm{A} \& \mathrm{E}$ department: drug used, dosage, route of administration.
Patients with significant head injuries, referrals from other hospitals, admissions without fractures (that is, nerve or tendon injuries), and patients with injuries over 12 hours old were not included.

The results of this audit were presented at a staff meeting. An analgesic protocol (figure) was then introduced and circulated through the department to all staff.

Over the following one month a further 100 consecutive referrals to fracture clinic and 100 orthopaedic admissions were then assessed using the same criteria.

\section{Results}

FRACTURE CLINIC REFERRALS

These were divided into four groups: forearm, lower leg/ankle, hand/foot, and others. The numbers of fractures assessed in the initial and repeat audit are shown in table 1 . The analgesia given is detailed in table 2 .

Fracture clinic referrals receiving unsatisfactory analgesia were reduced from $91 \%$ to $69 \%$, a difference of $22 \%$ ( $95 \%$ confidence interval $10 \cdot 9 \%$ to $33 \cdot 1 \%, \mathrm{P}<0.001)$.

ORTHOPAEDIC ADMISSIONS

These were also divided into four groups: neck of femur, forearm, lower leg/ankle, and others. The numbers of fractures assessed are given in table 3 and the analgesics used in table 4 .

Orthopaedic admissions receiving unsatisfactory analgesia were reduced from $39 \%$ to $22 \%$, a fall of $17 \%$ ( $95 \%$ confidence interval $4 \cdot 2 \%$ to $29 \cdot 8 \%, P=0.009$ ). The number of orthopaedic admissions receiving intravenous opiates increased by $28 \%$, from $9 \%$ to $37 \%$ (95\% confidence interval $16.3 \%$ to $39.7 \%$, $\mathbf{P}<0.001)$. The most appreciable improvements in analgesia used were obtained treating patients with fractured neck of femur (table 5).

\section{Discussion}

The management of pain in acute trauma is often neglected. ${ }^{12}$ Patients arriving at A\&E departments with acute trauma are unlikely to have received sufficient analgesia, ${ }^{3}$ so responsibility lies with the attending doctor.

Our study showed that before intervention many patients received no analgesia at all. The introduction of a protocol, however, significantly improved management. The protocol also led to more appropriate analgesic use. Intravenous analgesia is superior to intramuscular analgesia for reasons of speed of onset, reliability of uptake, and the ability to titrate doses to response. ${ }^{4-6}$ The number of orthopaedic admissions receiving intravenous 
opiates increased by $28 \%$ following the introduction of the protocol.

We were particularly successful in improving analgesia for patients with fractured neck of femur. Perhaps the value of intravenous titration of doses is better appreciated in the elderly population who typically suffer this injury.

In spite of this progress, many patients still receive no analgesia or inappropriate analgesia. The use of intramuscular diclofenac was unchanged by our protocol. Its use in acute trauma has not been fully assessed and it has no clear advantages over other forms of analgesia. ${ }^{4}$ Its use may owe more to its convenience to the physician than to proven efficacy for these injuries. When opiates are contraindicated, however, ketorolac may provide a useful alternative. ${ }^{6} 7$

Analgesia seems to be a neglected area in the management of acute trauma; few studies have been published and there is little evidence of

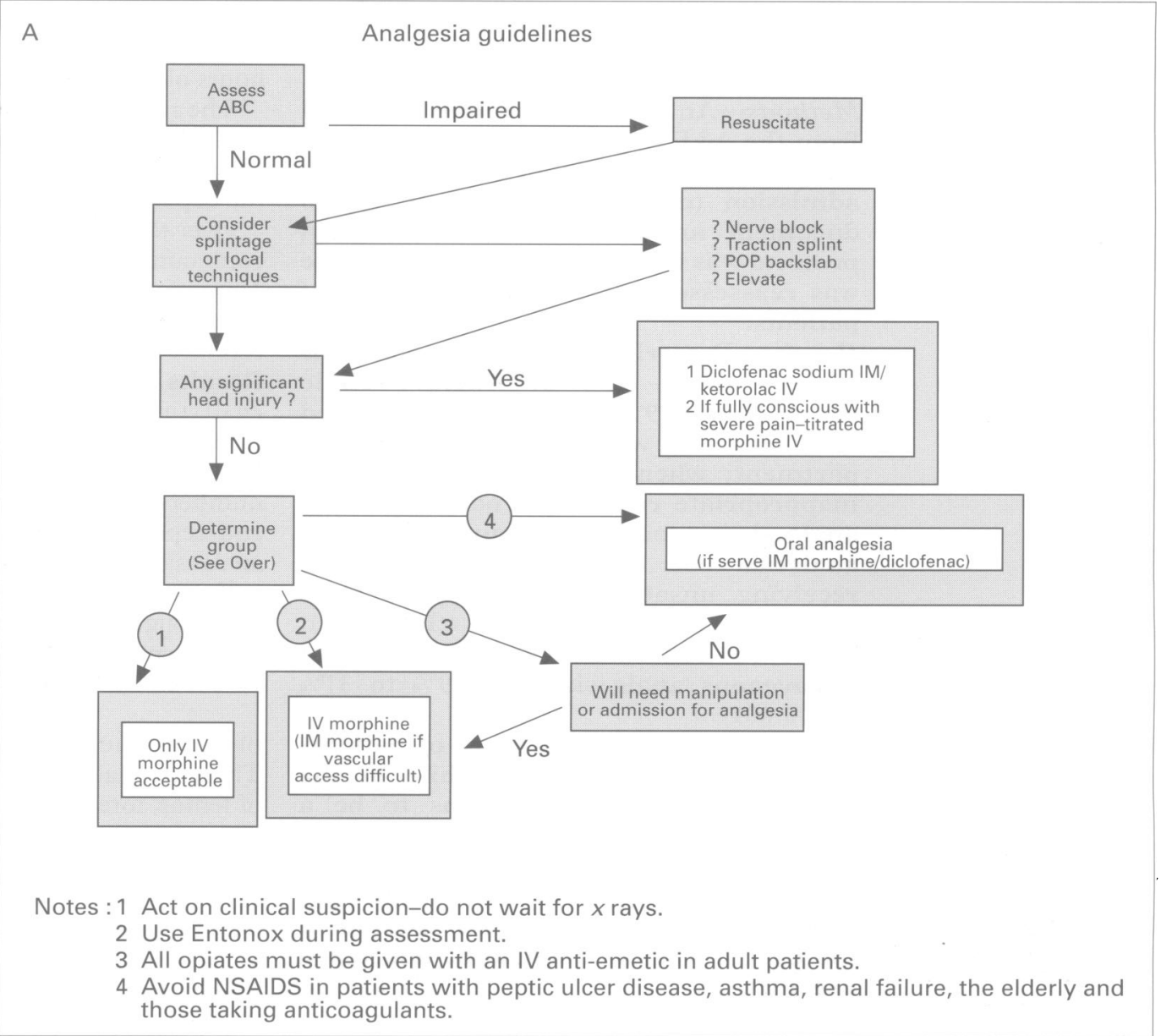

B

Group 1 Multiple trauma \# Femoral shaft

Unstable pelvic \#

Unstable spinal \#

Dislocated hip

\# acetabulum

\# Tib and fib

Dislocated elbow

Group 2 \# Neck of femur \# Tibial plateau Dislocated shoulder

Stable spinal \#

\# $>2$ ribs

\# humeral shaft
Group 3 \# Tibia or fibula Malleolar \# Tarsal/metatarsal \# Calcaneal \# Supracondylar \# elbow \# olecranon \# radius and/or ulna

Group 4 \# Neck of humerus \# Sternum \# clavicle

\# coccyx/sacrum Simple hand/foot \#s/dislocations \# Pubic ramus AC dislocation $\#<2$ ribs

Avulsion \# 
Table 1 Fracture clinic referrals

\begin{tabular}{lll}
\hline Fracture site & Initial audit & Repeat audit \\
\hline Forearm & 35 & 42 \\
Lower leg/ankle & 13 & 17 \\
Hand/foot & 35 & 17 \\
Others & 17 & 24 \\
\hline
\end{tabular}

Table 2 Analgesia given to fracture clinic referrals

\begin{tabular}{llc}
\hline & Initial audit & Repeat audit \\
\hline No analgesia offered & 91 & 69 \\
Declined analgesia & 0 & 4 \\
Paracetamol & 4 & 5 \\
Compound codeine-paracetamol & 1 & 4 \\
Oral diclofenac & 0 & 3 \\
IM diclofenac & 4 & 8 \\
IM opiate & 0 & 3 \\
IV opiate & 0 & 3 \\
Local anaesthetic block & 0 & 1 \\
\hline
\end{tabular}

IM, intramuscular; IV, intravenous.

Table 3 Orthopaedic admissions

\begin{tabular}{lll}
\hline & Initial audit & Repeat audit \\
\hline Neck of femur & 15 & 32 \\
Forearm & 32 & 18 \\
Lower leg/ankle & 17 & 17 \\
Others & 36 & 33 \\
\hline
\end{tabular}

Table 4 Analgesia given to orthopaedic admissions

\begin{tabular}{lcc}
\hline & Initial audit & Repeat audit \\
\hline No analgesia offered & 39 & 22 \\
Declined analgesia & 0 & 2 \\
Paracetamol & 1 & 2 \\
Oral diclofenac & 1 & 1 \\
IM diclofenac & 16 & 12 \\
IM opiate & 31 & 23 \\
IV opiate & 9 & 37 \\
Local anaesthetic block & 0 & 1 \\
Oral morphine & 3 & 0 \\
\hline
\end{tabular}

IM, intramuscular; IV, intravenous.

Table 5 Analgesia given to orthopaedic admissions with fractured neck of femur

\begin{tabular}{lcc}
\hline & Initial audit & Repeat audit \\
\hline No analgesia offered & $4(27 \%)$ & $2(6 \%)$ \\
IV opiate & 0 & $16(50 \%)$ \\
IM opiate & $8(53 \%)$ & $10(31.5 \%)$ \\
IM diclofenac & $3(20 \%)$ & $3(9 \cdot 5 \%)$ \\
Declined analgesia & 0 & $1(3 \%)$ \\
Total & 15 & 32 \\
\hline
\end{tabular}

IV, intravenous; IM, intramuscular.

audit. This is surprising, as the relief of pain is one of the principal interventions available to the emergency physician. Indeed, for many of the patients in our study it may be the only intervention made before referral for specialist care. In addition - as our study proves - the provision of analgesia is readily amenable to audit and as such provides a powerful indicator of the quality of medical care in A\&E. Perhaps this should be addressed by the Patients Charter.

It is evident that other countries and other specialties have given this subject more attention than our specialty in the United
Kingdom. Authoritative guidelines relating to analgesia in acute trauma have been published in the USA ${ }^{6}$ and Canada ${ }^{8}$ to act as a standard of care for patients. Although widely circulated medical publications ${ }^{4}{ }^{9}$ have approached the subject in the United Kingdom, as yet no coordinated guidelines or standards have been developed. Progress in other specialties is likewise more impressive; the problem of inadequate postoperative analgesia has long been recognised ${ }^{10}$ and innovative techniques produced to counter it, ${ }^{11}$ while the management of both chronic and cancer related pain is becoming a specialty in itself.

The reasons behind the neglect of analgesia are not clear. Ethnicity has been cited as a possible factor in determining analgesia use ${ }^{12}$ and children may also be denied adequate analgesia. ${ }^{13}$ There may be a latent period after injury before pain develops, which could be a factor in the assessment of analgesic requirements in $A \& E .{ }^{14}$ Clearly further work is required in this area. In the meantime the development of guidelines and standards of care by a nationally recognised organisation in $\mathrm{A} \& \mathrm{E}$ medicine should be addressed with urgency.

CONCLUSION

The use of audit and a protocol can improve the provision of analgesia in the $A \& E$ department when introduced locally. However, only limited improvements will be achieved until nationally recognised guidelines are developed. This important area of patient care deserves much more attention.

We thank Dr Liddy Goyder for help with statistical analysis

1 Wilson JE, Pendleton JM Oligoanalgesia in the emergency department. Am $\mathcal{F}$ Emerg Med 1989;7:620-3.

2 McKibben B, Irving M, Baskett PJF, Murley R. Saving lives: the NHS accident and emergency service and how to improve it. London: The LEA Health and Welfare Unit, 1991

3 Chambers JA, Guly HR. The need for better pre-hospital analgesia. Arch Emerg Med 1993;10:187-92.

4 Macrae WA, Davies HT. Pain from acute trauma. Prescribers f 1993;33:232-7.

5 Illingworth KA, Simpson KM. Anaesthesia and analgesia in emergency medicine. Oxford: Oxford University Press, 1994.

6 US Department of Health and Human Services. Acute pain management, operative or medical procedures and trauma. Clinical practice guidelines 1992. Rockville, Maryland: Agency for Health Care Policy and Research, 1992.
(AHCPR Publication No 92-0032.)

7 Buckley MM-T, Brogden RM. Ketorolac: a review of its pharmacodynamic and pharmacokinetic properties and pharmacodynamic and pharmacokinetic proper
therapeutic potential. Drugs 1990;39:86-109.

8 Ducharme J. Emergency pain managment: a Canadian Association of Emergency Physicians (CAEP) cosensus Association of Emergency Physicians (CAE

9 Managing acute pain in children. Drug Ther Bull 1995; 33(6).

10 Post-operative pain. $B M F$ 1978,ii:517.

11 Owen H, Mather LE, Rowley K. The development and clinical use of patient-controlled analgesia. Anaesth Intensive Care 1988;16:437-47.

12 Todd KH, Samaroo N, Hoffman JR. Ethnicity as a risk factor for inadequate emergency department analgesia. ҰAMA 1993;269:1537-9.

13 Selbst SM. Analgesia in children. Why is it underused in emergency departments? Drug safety 1992;7:8-13.

14 Melzack R, Wall P, Ty TC. Acute pain in an emergency clinic: latency of onset and descriptor patterns related to different injuries. Pain 1982;14:33-43. 